\title{
Teledentistry in a Dental Students' Examination - Assessing the Acceptance and Feasibility of a Virtual Dental Examination
}

\author{
Britz, Vanessa ${ }^{1,2}$; Sterz, Jasmina ${ }^{1,3}$; Ruesseler, Miriam ${ }^{1,3}$; Thoenissen, Phillip ${ }^{4}$; Sader, Robert ${ }^{4}$; \\ Seifert, Lukas Benedikt ${ }^{4}$ \\ ${ }^{1}$ Frankfurt Interdisciplinary Simulation Center FIneST, Medical Faculty, Goethe University, Frankfurt, Germany \\ ${ }^{2}$ Frankfurt Reference Centre for Rare Disease, University Hospital, Frankfurt, Goethe University, Frankfurt, \\ Germany \\ ${ }^{3}$ Department for Trauma-, Hand- and Reconstructive Surgery, University Hospital Frankfurt, Goethe University, \\ Frankfurt, Germany \\ ${ }^{4}$ Department of Oral, Cranio-Maxillofacial, and Facial Plastic Surgery, University Hospital Frankfurt, Goethe \\ University, Frankfurt, Germany \\ Email: miriam.ruesseler@kgu.de
}

\begin{abstract}
Due to the coronavirus pandemia, dental schools are facing a change onto virtual solutions. Teledentistry has proven to be an opportunity to bridge this gap. This study aims to assess the feasibility and acceptance of a virtual dental examination.

It took place during the final maxillofacial examination. Study participants were dental students and real inpatients. A video-call-software was used to carry out the examination. To assess the acceptance participants filled out a questionnaire afterwards.

22 patients answered the questionnaire. They didn't 'feel inhibited' and had no 'difficulties developing a relationship with the student'. 28 students answered the questionnaire. They found the conversation at eye level but expressed more inhibitions than the patients in a virtual setting, still finding themselves able to have an adequate doctor-patient conversation.

Telemedicine seems well accepted on patients' and students' side, offering a safe alternative to enable students to maintain dental teaching in a pandemic situation.
\end{abstract}

Keywords: dental examination; teledentistry; virtual examination; Covid-19; undergraduate education.

\section{Introduction}

Due to the coronavirus disease pandemic main parts of medical education had to be set on hold. The necessity of limiting student's direct patient encounters down to a minimum, as potential victims and carriers for the virus, has a tremendous effect on medical education (Tabatabai, 2020).

Especially dental professionals and practical dental school education, which invariably involves close inspection, examination, diagnostic and therapeutical interventions of the naso-oro-pharyngeal region, are most susceptible to get infected with corona virus (Peng et al., 2020).

Therefore, dental schools are facing an inevitable change from the old classroom onto virtual solutions (Chavarría-Bolaños, Gómez-Fernández, Dittel-Jiménez, \& Montero-Aguilar, 2020). Undoubtedly, direct in-person patient contact has an important educational value to medical and dental students. Although there can't be an exact replacement, telemedicine has proven to be a unique opportunity to bridge this current gap (Iancu, Kemp, \& Alam, 2020).

Teledentistry as a subunit of telemedicine is found to be comparable to real time consultation in areas with limited access to facilities, in school children and in long-term-healthcare facilities (Ghai, 2020). Teleconsultation and telediagnosis have therefore been rated positively and beneficial for dental care (Ghai, 2020). In terms of medical school training and examinations, telemedicine has already found its application in zero-patient contact virtual practical exit examinations for orthopedic residents (Malhotra, Gautam, George, Goyal, \& Ansari, 2020) or running virtual OSCE cases (Hopwood, Myers, \& Sturrock, 2020; Sartori, Hayes, Horlick, Adams, \& Zabar, 2020; Sartori, Olsen, Weinshel, \& Zabar, 2019). For dental schools the virtualization process is an ongoing progress, although it was abruptly imposed, but 
also represents an opportunity to assess and classify dental curricula and their exams for virtualization (Chavarría-Bolaños, Gómez-Fernández, Dittel-Jiménez, \& Montero-Aguilar, 2020).

Therefore, the present study aims to assess the feasibility and acceptance of a virtual dental examination for last year dental students and their patients.

\section{Material and Methods}

\subsection{Study Population and Background}

This prospective study took place during the final maxillofacial examination. Study participants were last year dental students who had to complete this examination as part of their curricular dental training.

Participating patients were real inpatients of the University Hospital, Clinic for Oral-, CranioMaxillofacial and Plastic Surgery, no Simulated Patients (Atkins, Roberts, Hawthorne, \& Greenhalgh, 2016). Prerequisite for patient's participation was being well grounded in German language. There were no further inclusion or exclusion criteria. Participation in the study was voluntary for both, students and patients, and took place after written informed consent.

The study was conducted according to ethical principles of the Helsinki Declaration (Ethical Principles for Medical Research Involving Human Subjects). Since the present study only involved an evaluation and was not considered a human research proposal, no ethics vote was requisite according to the guidelines of the ethics committee of the University Hospital of Frankfurt.

\subsection{Examination}

Without pandemic restrictions on the first day of the two-day-long lasting examination each student would be assigned a patient on the ward, which they have to take a medical history from and perform a symptom orientated facial and oral examination. A written summary of the patient's history would have to be submitted on the same day. On the second day students would perform an oral examination.

Due to the pandemic situation the examination couldn't take place under normal conditions. Therefore, on the first day students and patients were using a video-call-software to carry out the anamnesis interview, without having to be in the same room. In this case a secure connection was granted via Cisco Webex Meetings (Cisco Webex, Milpitas, California), which is authorized by the University Hospital for the transmission of sensitive data. An intern invigilated the examination and established the connection between student and patient. In order to ensure the same examination conditions for all students, they were alone in a low-irritation room during the conversation. A student assistant was present to help with technical problems.

The patient's history was taken completely via video-call. Examination results were evaluated via video-call as well, as far as possible. Additionally, intraoral examination images of the patients were shown to the students. On average the call-duration lasted between 25-30 minutes, however, there was no set time limit.

The feasibility and acceptance evaluation were solely performed on day one, as day two could be performed without restrictions.

\subsection{Questionnaire}

Prior to the examination two questionnaires (one for the patients and one for the students) were designed and concerted by an expert group based on a literature review. The items were tested regarding comprehensibility by a group of medical education specialists who were not involved in designing the questionnaires. Based on the answers of the medical education specialists the questionnaires were edited. Each questionnaire consists of 13 questions, 7 of which were concurrent for both groups. The remaining 6 questions were group specific.

The questions were answered by both groups after the exam by using a 5 point-Likert-scale $((1)$ Strongly agree; (2) Agree; (3) Neither agree nor disagree; (4) Disagree; (5) Strongly disagree). 


\section{Results}

Table 1. Patients' responses in absolute numbers; concurrent questions in both groups are highlighted in grey. $\dagger=$ Average, $\ddagger=$ Median, $\S=$ Standard Deviation

\begin{tabular}{llllllllll}
\hline Questions & $\mathbf{1}$ & $\mathbf{2}$ & $\mathbf{3}$ & $\mathbf{4}$ & $\mathbf{5}$ & $\mathbf{n}$ & $\mathbf{A v g}^{\dagger}$ & $\mathbf{M e d}^{\ddagger}$ & $\mathbf{S D}^{\S}$ \\
\hline I felt that I was being well looked after. & 15 & 5 & 1 & 1 & 0 & 22 & 1.48 & 1 & 0.79 \\
\hline We had a pleasant conversation. & 17 & 5 & 0 & 0 & 0 & 22 & 1.45 & 1 & 0.42 \\
\hline The student understood well what was important to me. & 13 & 8 & 1 & 0 & 0 & 22 & 1.45 & 1 & 0.58 \\
\hline Too much time was spent making small talk. & 0 & 1 & 1 & 3 & 17 & 22 & 4.64 & 5 & 0.77 \\
\hline It was difficult to ask your own questions. & 2 & 2 & 2 & 6 & 10 & 22 & 3.91 & 4 & 1.31 \\
\hline A conversation took place at eye level. & 10 & 11 & 1 & 0 & 0 & 22 & 1.59 & 2 & 0.58 \\
\hline It was difficult to develop a relationship with the student. & 0 & 0 & 2 & 5 & 15 & 22 & 4.59 & 5 & 0.65 \\
\hline The student understood my feelings / emotional situation. & 12 & 8 & 1 & 1 & 0 & 22 & 1.59 & 1 & 0.78 \\
\hline The student saw things from my point of view. & 11 & 7 & 3 & 1 & 0 & 22 & 1.73 & 1.5 & 0.86 \\
\hline The student took my personal experience into account. & 10 & 8 & 2 & 1 & 0 & 22 & 1.71 & 2 & 0.82 \\
\hline $\begin{array}{l}\text { An adequate doctor-patient conversation was possible } \\
\text { when using telemedicine. }\end{array}$ & 12 & 6 & 4 & 0 & 0 & 22 & 1.64 & 1 & 0.77 \\
\hline $\begin{array}{l}\text { The doctor-patient conversation was possible without } \\
\text { interference. }\end{array}$ & 14 & 5 & 3 & 0 & 0 & 22 & 1.50 & 1 & 0.72 \\
\hline Using a computer made me feel inhibited. & 0 & 0 & 1 & 3 & 18 & 22 & 4.77 & 5 & 0.52 \\
\hline
\end{tabular}

Table 2. Students' responses in absolute numbers; concurrent questions in both groups are highlighted in grey. $\dagger=$ Average, $\ddagger=$ Median, $\S=$ Standard Deviation

\begin{tabular}{|c|c|c|c|c|c|c|c|c|c|}
\hline Questions & 1 & 2 & 3 & 4 & 5 & $\mathrm{n}$ & $\operatorname{Avg}^{\dagger}$ & Med $^{\ddagger}$ & $\mathrm{SD}^{\S}$ \\
\hline I felt that I was being well looked after. & 10 & 16 & 2 & 0 & 0 & 28 & 1.72 & 2 & 0.59 \\
\hline We had a pleasant conversation. & 21 & 6 & 1 & 0 & 0 & 28 & 1.29 & 1 & 0.52 \\
\hline $\begin{array}{l}\text { I was able to convey to the patient what was important to } \\
\text { me. }\end{array}$ & 11 & 15 & 1 & 1 & 0 & 28 & 1.71 & 2 & 0.7 \\
\hline Too much time was spent making small talk. & 0 & 0 & 4 & 9 & 15 & 28 & 4.39 & 5 & 0.72 \\
\hline It was difficult to ask the patient questions. & 0 & 2 & 3 & 10 & 13 & 28 & 4.21 & 4 & 0.90 \\
\hline A conversation took place at eye level. & 17 & 9 & 1 & 1 & 0 & 28 & 1.50 & 1 & 0.73 \\
\hline It was difficult to develop a relationship with the patient. & 0 & 1 & 5 & 10 & 12 & 28 & 4.81 & 4 & 0.85 \\
\hline $\begin{array}{l}\text { I was able to respond well to the patient's feelings / } \\
\text { emotional situation. }\end{array}$ & 7 & 14 & 6 & 1 & 0 & 28 & 2.04 & 2 & 0.78 \\
\hline I was able to respond well to the patient's perspective. & 4 & 19 & 4 & 1 & 0 & 28 & 2.07 & 2 & 0.65 \\
\hline $\begin{array}{l}\text { I was able to take into account the patient's personal } \\
\text { experience. }\end{array}$ & 4 & 19 & 2 & 3 & 0 & 28 & 2.14 & 2 & 0.79 \\
\hline $\begin{array}{l}\text { An adequate doctor-patient conversation was possible } \\
\text { when using telemedicine. }\end{array}$ & 7 & 13 & 6 & 2 & 0 & 28 & 2.11 & 2 & 0.86 \\
\hline $\begin{array}{l}\text { The doctor-patient conversation was possible without } \\
\text { interference. }\end{array}$ & 13 & 8 & 2 & 4 & 1 & 28 & 2.00 & 2 & 1.20 \\
\hline Using a computer made me feel inhibited. & 0 & 1 & 6 & 10 & 11 & 28 & 4.11 & 4 & 0.86 \\
\hline
\end{tabular}

\subsection{Patient Questionnaire}

The patient questionnaire was answered by 22 patients out of 30 . None of them were questioned by the same student. The mean age of participating patients was 42.2 years $( \pm 20,8)$. The present clinical pictures were trauma $(n=12)$, odontogenic infections $(n=5)$, jaw cysts $(n=2)$ and squamous epithelium carcinoma $(n=3)$. The results are displayed in Table 1.

It is noticeable that the patients didn't 'feel inhibited' due to a virtual setting $(4.77 \pm 0.52)$ and in the majority had no 'difficulties developing a relationship with the student' $(4.59 \pm 0.65)$. They mostly 
still felt well looked after $(1.48 \pm 0.79)$ and rated the conversation as 'on eye level' (1.59 \pm 0.58$)$. They also majoritarianly agreed that they were able to express their emotions and feelings making their counterpart understand what was important to them $(1.59 \pm 0.78)$.

\subsection{Student Questionnaire}

The student questionnaire was answered by 28 students out of 30.19 students were female, 9 male. The mean age was $23.5( \pm 1.7)$. The results are displayed in Table 2.

Similar to the patients' answers also the students found the conversation on eye level $(1.50 \pm 0.73)$ but expressed more inhibitions than the patients in a virtual setting $(4.11 \pm 0.86)$. In the majority they still found themselves able to have an adequate doctor-patient conversation $(2.11 \pm 0.86)$, which allowed them to reduce 'difficulties in developing a relationship with their patient $(4.81 \pm 0.85)$. They also mostly used positive ratings for "being able to respond well to the patient's feelings $(2.04 \pm 0.78)$ and perspective" $(2.07 \pm 0.65)$.

\section{Discussion}

The present study shows an overall high acceptance of a virtual solution for performing a dental examination.

Harendza et al. described in a virtual simulated patient-based training for final year students, that with respect to the telemedicine format, participants were glad an option had been found and was offered during the pandemic situation (Harendza, Gartner, Zelesniack, \& Prediger, 2020).

It is worth pointing out that no simulated patients were used for this setting but real patients, which allows a much more genuine evaluation of the students' acceptance. A review of sociolinguistic approaches to simulations demonstrates that simulated assessment, even if being 'realistic', shows some crucial differences compared to the communicative competences found in real-life practice (Atkins, Roberts, Hawthorne, \& Greenhalgh, 2016). In simulated consultations, candidates need to manage the fact of an inverted power relation, because knowledge and judgment rest with the simulated patient rather than with the physician student (Hanna \& Fins, 2006). This normally means an additional stressor in an already emotionally loaded exam situation. In this situation the students encountered real patients, which surely contributed to a high acceptance on the students' side.

According to a recent systematic review, barriers or challenges reported for implementing virtual solutions, is among others a high level of anxiety and stress experienced by the student (Regmi \& Jones, 2020). The examination situation might contribute to the higher level of inhibition expressed by the students compared to the responses of the patients.

Both sides stated the possibility of an adequate doctor-patient conversation using telemedicine. According to studies, medical personnel inexperienced with telemedicine often struggles with gaining detailed histories and find it difficult to translate their traditional interview and physical exam skills to the virtual sphere (Iancu, Kemp, \& Alam, 2020). Students in this study were also unexperienced to a virtual exam format, however, they were capable of translating their skills to a virtual setting.

Additionally, using intraoral examination images as supplements helps reducing the need of close clinical examination but still allows good clinical decision making on differential diagnosis for example in judging oral lesions (Machado, de Souza, Oliveira, Martelli Junior, \& Bonan, 2020; Maret, Peters, Vaysse, \& Vigarios, 2020).

However, the patient acceptance is the key to success of any module. Lack of face-to-face communication may lead to apprehension of patients, leading to inadequacy of proper communication of medical problems. In this setting the patient expressed rather rarely problems with the virtual setting which confirms findings of many surveys that teledentistry is gradually gaining acceptance by patients as well as healthcare providers (Estai et al., 2017; Petcu, Kimble, Ologeanu-Taddei, Bourdon, \& Giraudeau, 2017).

One also needs to discuss the fact, that since the patients knew about the examination situation of the students this could, even unconsciously, lead to more benevolent answers and therefore resemble a possible bias. 
A prior analysis of the personality structure of participating students was not assessable due to the short amount of time till the examination. However, personality structure plays an important role for the satisfaction of simulated patients. In particular simulated patients were more satisfied with students' communication when students were both good at recognizing nonverbally communicated emotions and scored high on extraversion (Schreckenbach et al., 2018), which is likely to be applicable for real patients as well and therefore highlights an interesting aspect. Since almost all students of one semester were included in this study, one can assume this represents a valid cross section of different personality types. Therefore, this possible bias seems negligible.

The chosen setting proved to be feasible with no additional personnel or time resources compared to a non-pandemic setting, provided that students and patients have access to a web-enabled video-telephony device.

Mentionable limitations of this study are the small perspective on one medical field within one faculty. There is no comparable data of a comparison group under non-pandemic conditions, however, with the participation of almost one whole semester the answers of the questionnaires represent a valid cross section.

\section{Conclusion}

Telemedicine is feasible and seems overall well accepted on patients' and students' side for a dental examination, offering a safe alternative to enable students to complete their dental training during the Covid-19 pandemic. It is also a solution to maintain medical and dental teaching and examination without endangering patients and students in a pandemic situation. To overcome still existing inhibitions with the technology dentist and dental students should be trained and educated adequately about teledentistry to increase the acceptance even further, probably implementing e-learning and telemedicine earlier in dental curricula.

Acknowledgements. The authors would like to thank students and patients for their participation.

\section{References}

1. S. Atkins, C. Roberts, K. Hawthorne, and T. Greenhalgh (2016), "Simulated consultations: a sociolinguistic perspective," BMC Medical Education, vol. 16, p. 16

2. D. Chavarría-Bolaños, A. Gómez-Fernández, C. Dittel-Jiménez, and M. Montero-Aguilar (2020), "E-Learning in Dental Schools in the Times of COVID-19: A Review and Analysis of an Educational Resource in Times of the COVID-19 Pandemic," Odovtos International Journal of Dental Sciences, vol. 22, pp. 69-86.

3. M. Estai, Y. Kanagasingam, D. Xiao, J. Vignarajan, S. Bunt, E. Kruger, and M. Tennant (2017), "End-user acceptance of a cloud-based teledentistry system and Android phone app for remote screening for oral diseases," Journal of Telemedicine and Telecare, vol. 23 no. 1, pp. 44-52.

4. S. Ghai (2020), "Teledentistry during COVID-19 pandemic," Diabetes and Metabolic Syndrome, vol. 14 no. 5 , pp. 933-935.

5. M. Hanna, and J. J. Fins (2006), "Viewpoint: power and communication: why simulation training ought to be complemented by experiential and humanist learning," Academic Medicine, vol. 81 no. 3, pp. 265-270.

6. S. Harendza, J. Gartner, E. Zelesniack, and S. Prediger (2020), "Evaluation of a telemedicine-based training for final-year medical students including simulated patient consultations, documentation, and case presentation," GMS Journal for Medical Education, vol. 37 no. 7, p. Doc94.

7. J. Hopwood, G. Myers, and A. Sturrock (2020), "Twelve tips for conducting a virtual OSCE," Medical Teacher, pp. 1-4.

A. M. Iancu, M. T. Kemp, and H. B. Alam (2020), "Unmuting Medical Students' Education: Utilizing Telemedicine During the COVID-19 Pandemic and Beyond," Journal of Medical Internet Research, vol. 22 no. 7, p. e19667.

8. R. A. Machado, N. L. de Souza, R. M. Oliveira, H. Martelli Junior, and P. R. F. Bonan (2020), "Social media and telemedicine for oral diagnosis and counselling in the COVID-19 era," Oral Oncology, vol. 105 n, p. 104685. 
9. R. Malhotra, D. Gautam, J. George, D. Goyal, and M. T. Ansari (2020), "Conducting orthopaedic practical examination during the Covid-19 pandemic," Journal of Clinical Orthopaedics and Trauma, vol. 11, Suppl 4, pp. S448-S455

10. D. Maret, O. A. Peters, F. Vaysse, and E. Vigarios (2020), "Integration of telemedicine into the public health response to COVID-19 must include dentists," International Endodontic Journal, vol. 53 no. 6, pp. 880-881.

11. X. Peng, X. Xu, Y. Li, L. Cheng, X. Zhou, and B. Ren (2020), "Transmission routes of 2019-nCoV and controls in dental practice," International Journal of Oral Science, vol. 12 no. 1, p. 9.

12. R. Petcu, C. Kimble, R. Ologeanu-Taddei, I. Bourdon, and N. Giraudeau (2017), "Assessing Patient's Perception of Oral Teleconsultation," International Journal of Technology Assessment in Health Care, vol. 33 no. 2, pp. 147-154.

13. K. Regmi, and L. Jones (2020), "A systematic review of the factors - enablers and barriers - affecting e-learning in health sciences education," BMC Medical Education, vol. 20 no. 1, p. 91.

14. D. J. Sartori, R. W. Hayes, M. Horlick, J. G. Adams, and S. R. Zabar (2020), "The TeleHealth OSCE: Preparing Trainees to Use Telemedicine as a Tool for Transitions of Care," The Journal of Graduate Medical Education, vol. 12 no. 6, pp. 764-768.

15. D. J. Sartori, S. Olsen, E. Weinshel, and S. R. Zabar (2019), "Preparing trainees for telemedicine: a virtual OSCE pilot," Medical Education, vol. 53 no. 5, pp. 517-518.

16. T. Schreckenbach, F. Ochsendorf, J. Sterz, M. Russeler, W. O. Bechstein, B. Bender, and M. N. Bechtoldt (2018), "Emotion recognition and extraversion of medical students interact to predict their empathic communication perceived by simulated patients," BMC Medical Education, vol. 18 no. 1, p. 237.

17. S. Tabatabai (2020), "COVID-19 impact and virtual medical education," Journal of Advances in Medical Education \& Professionalism, vol. 8 no. 3, pp. 140-143. 